Review Article

\title{
Electrochromic Glazing
}

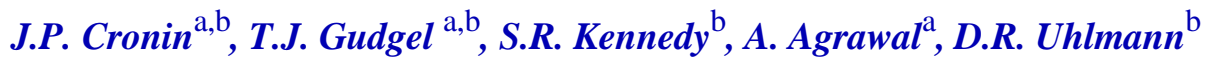 \\ ${ }^{\mathrm{a}}$ Advanced Technology Center, \\ Donnelly Corporation, \\ 4545 East Fort Lowell Road, Tucson, AZ 85712, USA \\ e-mail:john@atcdonnelly.com \\ ${ }^{\mathrm{b}}$ Department of Materials Science \& Engineering, \\ University of Arizona, \\ 4715 East Fort Lowell Rd., Tucson, AZ 85712, USA
}

Received: August 31, 1998; Revised: November 27, 1998

\begin{abstract}
Commercialization of large-area electrochromic glazing is reviewed with respect to device configuration and materials employed. Device configurations including electrochromic solutions and solid state electrochromic layers are discussed as well as novel user-controlled photochromic and gasochromic devices.
\end{abstract}

Keywords: electrochromic, photochromic, device, materials, review

\section{Introduction}

Over the past ten years there has been a dramatic increase in electrochromic (EC) research activity, as seen in the large number of scientific papers and patents issued during this time. This surge in research is driven by the need to produce more energy efficient glazings which can lead to a reduction in fossil fuel consumption. The primary advantage of an EC glazing is the capability for dynamic control of the transmissive properties of the glass. Such windows can be adjusted to reduce glare and daylight heating and cooling needs or to provide nighttime insulation and privacy. A comprehensive study on the economic benefits of electrochromic windows determined that they provide greater energy savings than static-control glasses ${ }^{1}$. EC technology was found to reduce overall electricity demands by taking advantage of daylight during certain times of the day. Ideally one would like EC glazings especially for architectural use to reject the maximum possible levels of near-IR radiation during the summer to lower air-conditioning costs but pass as much near-IR as possible during the winter months to reduce heating costs.

Many industrial institutions are investigating EC devices but relatively few products are commercially avail$a_{b l e}^{2}$. The most successful applications to date have been rear-view mirrors for automobiles. Several million of these mirrors have been produced over the past five years. However, EC devices for these applications are small in size compared to most glazing requirements. Considerable activity is taking place to expand EC technology to larger area applications such as automotive and architectural glazing. This is not an easy task since increasing the size of an EC device involves the combination of many different areas of research which are all interdependent. The task is to combine all of these desired changes into a large-area, singledevice configuration.

The main areas envisioned for usage of EC glazings are automotive and architectural, with automotive being the most likely entry point mainly because of its smaller size requirements. However, frontal automotive glazing must be $70 \%$ transmitting weighted to Illuminant A to ensure glazing is adequately transmitting for night driving and the windows must be safety glazed ${ }^{3}$. For most architectural applications these restrictions do not apply but the size requirements are much greater than those for automotive applications. Sunroofs are most likely to be the first application of EC glazing in automobiles. They have the advantage in that because of their location in a vehicle they do not have to comply with the high transmission requirements and they are relatively small, in typical sizes less than 0.7 
Table 1.

\begin{tabular}{lcc}
\hline Requirement & Automotive Glazing & Architectural Glazing \\
\hline Lifetime & Decade & Decades \\
Type of Window & Compound curvature & Typically flat \\
Bleached \% & $70 \% \mathrm{~T}$ (Photopic) or less & Can be $70 \% \mathrm{~T}$ (Photopic) \\
Temperature range & -40 to $100^{\circ} \mathrm{C}$ & -40 to $85^{\circ} \mathrm{C}$ \\
Response time & Several minutes to seconds & Several minutes \\
Desired EC Configuration & Solid State & Solid State \\
\hline
\end{tabular}

square meters. The different requirements for automotive and architectural glazing are listed in the Table 1.

\section{Recent Electrochromic Device Technologies Available}

Several device technologies are currently available for architectural and automotive glazing applications. Much of the device work today falls into a small number of basic designs. If one looks to the recently issued patent literature as an indicator of the technologies which are currently being pursued for commercial applications, examples of most of these basic designs can be found. It is worthwhile, therefore, to discuss some of these designs in terms of their basic function and composition as well as some of the advantages and disadvantages attributed to these designs.

\subsection{Solution phase electrochromics}

Many of the early electrochromic devices derived their optical modulation from redox reactions in organic solutions. The general construction of such a device is depicted in Fig. 1. The two glass substrates with their associated transparent conductors form a cavity which is filled with an electrolyte solution. An electrical potential applied to the transparent conductors causes a set of reversible electrochemical reactions within the electrolyte which cause the change in transmission of the device.

The electrolyte contains at least two redox species which are generally colorless in solution in the absence of electrical perturbation. Under the application of a potential between the transparent conductors, one component of the electrolyte is reduced while another component is oxidized. Using subscripts to refer to the redox species in the electrolyte, the pertinent chemical reactions occurring on the transparent electrodes under application of an applied DC potential are as follows:

$$
\begin{aligned}
& \mathrm{Ox}_{1}(\text { colorless })+\mathrm{n} \mathrm{e}^{-} \rightleftharpoons \operatorname{Red}_{1}(\text { colored }) \\
& \operatorname{Red}_{2}(\text { colorless }) \rightleftharpoons \mathrm{Ox}_{2}(\text { colored })+\mathrm{ne}^{-}
\end{aligned}
$$

These reactions occur at the cathode and anode respectively within the device. The species are chosen such that the equilibrium in the absence of an applied potential lies

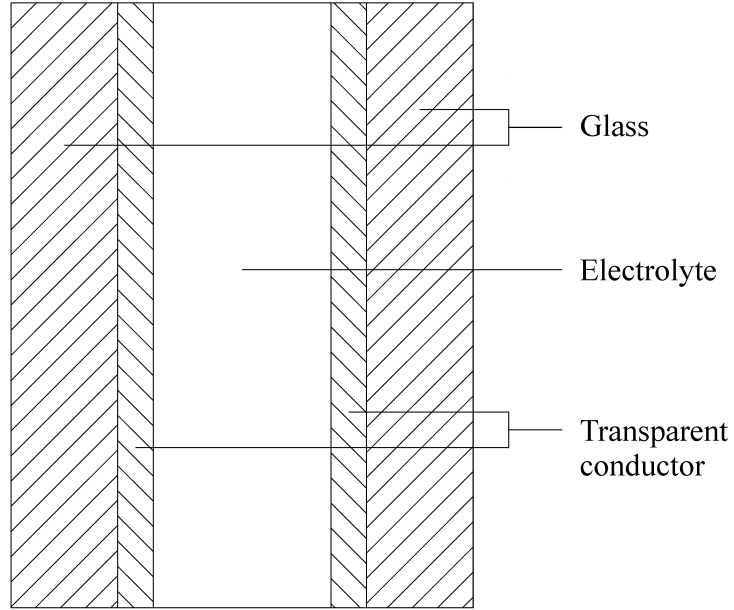

Figure 1.

completely to the left in the above reactions. Thus, the species $\mathrm{Red}_{1}$ and $\mathrm{Ox}_{2}$ can react in the solution to reform their lower energy, colorless forms $\mathrm{Ox}_{1}$ and $\mathrm{Red}_{2}$. In the absence of an applied potential, this recombination of the colored species to form the colorless species results in a spontaneous bleaching of the device. Due to their selfbleaching nature and their relatively simple construction, they have proven viable in such applications as automotive rearview mirrors ${ }^{3}$.

The recent patent literature has outlined some of the difficulties surrounding the commercialization of these devices. While the self-bleach mechanism is a feature for some applications, it necessarily implies that a sustaining current be applied to the device to maintain a desired degree of coloration. In an application such as architectural glazing, this self-bleach mechanism is not necessary, and its inhibition is desirable to reduce the power consumption in the glazing. This issue is currently being addressed. For example, Varaprasad ${ }^{4}$ has found certain combinations of solvents and coloring species which lead to sizable reductions in current consumption.

Stability under UV radiation has also been an issue, and several recent patents have been issued dealing at least in 
part with addition of stabilizers to the electrolytes to reduce the UV degradation ${ }^{4,5}$.

An effect common to devices of this type is a banding of coloration near the electrical contacts to the transparent conductors after prolonged coloration. This phenomenon has been termed segregation, and is due to migration and convection of the anodic and cathodic coloring species within the electrolyte. Generally, this effect can be reduced by addition of thickeners to electrolytes of high ionic conductivity and using decreased concentrations of the coloring species ${ }^{6,4}$.

\subsection{Electrochromic devices incorporating solid electrochromic films}

A great many electrochromic devices rely on the coloration of solid films to effect the change in optical modulation of the device. Such electrochromic films are broadly classed into anodic (coloring upon electrochemical oxidation) and cathodic (coloring upon electrochemical reduction) coloring films. The reactions resulting in the coloration are generally summarized as follows:

Anodic materials such as nickel oxyhydroxide:

$$
\text { (transparent) } \mathrm{Ni}(\mathrm{OH})_{2} \rightleftharpoons \begin{aligned}
& \mathrm{NiO}_{\mathrm{x}}(\mathrm{OH})_{2-\mathrm{x}} \\
& (\text { colored })+\mathrm{xH}^{+}+\mathrm{xe}^{-}
\end{aligned}
$$

Cathodic materials such as tungsten oxide:

$$
\text { (transparent) } \mathrm{WO}_{3}+\mathrm{ne}^{-}+\mathrm{nM}^{+} \rightleftharpoons \begin{aligned}
& \mathrm{M}_{\mathrm{n}} \mathrm{WO}_{3} \\
& \text { (colored) }
\end{aligned}
$$$$
\left(\mathrm{M}=\mathrm{H}^{+}, \mathrm{Li}^{+}, \mathrm{Na}^{+}\right)
$$

Since the electrochemical reactions involve the insertion and removal of cations into and out of the solid films, another component is required in such devices to act as a sink for the cations and electrons upon removal from the electrochromic film. There are primarily three ways this has been reduced to practice - ion storage layers, coupling of anodic and cathodic electrochromic materials, and incorporation of redox promoters in the electrolyte. Each of these will be discussed in turn.

\subsubsection{Ion storage layer devices}

Devices incorporating ion storage layers generally follow the construction shown in Fig. 2. The two faces of the device generally consist of transparent conductive oxide coated glass substrates opposing one another with a gap in between forming a cavity. An electrochromic material such as tungsten oxide is coated on the interior face of one electrode. The opposing electrode is coated with a layer which is capable of reversible insertion of the cations and electrons upon removal from the electrochromic layer. A suitable electrolyte with low electronic conductivity and high ionic conductivity is then placed between the electro-

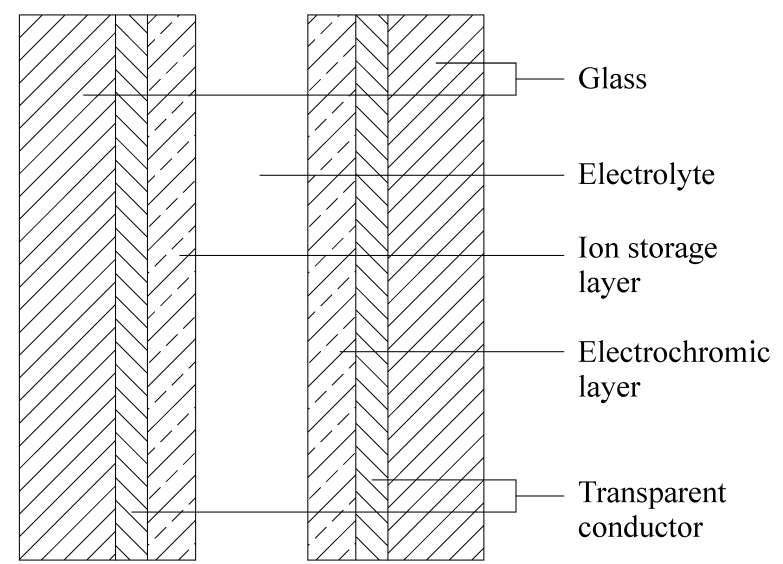

Figure 2.

chromic and ion storage layers. Upon application of an appropriate DC potential between the conductive electrodes, the cations and electrons can be inserted into the electrochromic electrode causing coloration. Upon reversal of this applied potential, the cations are shuttled through the electrolyte and into the ion storage layer where they are compensated by electrons transported from the electrochromic layer via an external circuit. In this manner, the optical modulation can be adjusted reversibly by application of appropriate DC potentials.

One of the attractive features of these devices is their capability for color memory (retaining a selected level of transmission after removal of the applied potential). While the construction of such devices may be more difficult than that of the solution phase devices due to the deposition of the multiple thin films, this does not appear to have discouraged research in this area. Several recent patents on these device structures indicates that commercial interest remains e.g. ${ }^{7,8,9}$.

The absence of a rapid self-bleach in many of these devices could explain the exclusion of such structures in the present commercialized applications such as automotive mirrors. This hurdle has been addressed by imposing a low level of electrical conductivity in the materials used to seal the device ${ }^{10}$. Since this sealing material contacts both transparent conductors, it acts as a weak short which results in a self-bleaching behavior in the event of electrical failure to the device, and it may open the door for these devices in applications requiring such a safety feature.

The extent of memory possible with these devices may prove advantageous when scaling to large-area applications with the effect of reducing the power requirements. However, the complexity of depositing the many layers often associated with these devices could result in decreased yields and greater expense for their production. 


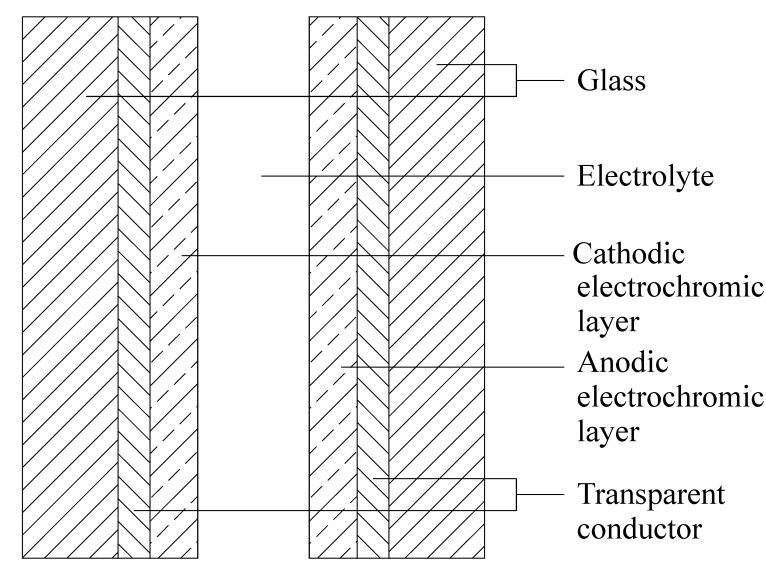

Figure 3.

\subsubsection{Devices containing both anodic and cathodic electrochromic layers}

These devices can be considered to be a subset of the previously described group containing ion storage layers. A typical construction is described by Nagai ${ }^{11}$, and Fig. 3 shows their similarity to the ion storage devices. In this case, the ion storage layer is replaced by a coloring electrochromic layer which colors in a state complementary to the electrochromic layer. For example, if one electrochromic layer is chosen to be tungsten oxide (an electrochromic material which exhibits cathodic coloration), an appropriate choice for the other electrochromic layer might be nickel oxide (an electrochromic material which exhibits anodic coloration). Upon application of a DC potential to the device, one electrochromic layer is reduced while the other is oxidized. Since tungsten oxide is colored in the reduced state and nickel oxide is colored in the oxidized state, complementary coloration is observed which results in effective use of both electrochromic films to obtain the desired level of optical modulation. Likewise, when the tungsten oxide is in the oxidized state, the nickel oxide is reduced resulting in a device with a high level of transmission.

While these devices are perhaps more efficient in their utilization of charge in the device, they have similar attributes to the devices incorporating ion storage layers. While many ion storage layers exhibit mild electrochromic activity, there is a diminished set of materials which exhibit strong electrochromic activity for use specifically in these devices. Perhaps it is for these reasons that the literature contains lesser examples of such devices despite their conceptual advantages.

\subsubsection{Electrochromic devices incorporating solution} phase redox promoters

Another method of compensating the cations and electrons upon withdrawal from the electrochromic film is

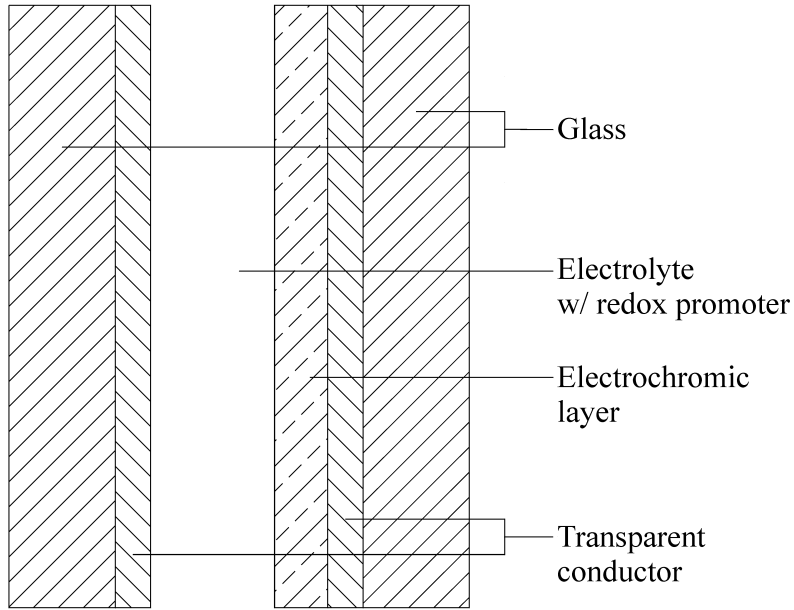

Figure 4.

based on solution phase redox promoters. A typical construction is depicted in Fig. 4. Two opposing glass substrates whose inner surface is coated with a transparent conductive film again form the basis for the device. An electrochromic film is coated onto one of the conductors, and the other conductor is left bare. An electrolyte with one or more redox promoters is then disposed between the electrochromic film and the transparent conductor of the opposing electrode.

The redox promoter serves as the source and sink for electrons used for the electrochemical reduction and oxidation of the electrochromic film. One of the first redox promoters found in the patent literature examples for electrochromic devices is iodine which undergoes the reversible iodide/triiodide reaction (another example of redox promoters involves the combination of a metallocene and a phenothiazine ${ }^{12}$ ). One example from Kamimori ${ }^{13}$ describes the addition of the redox promoter in the form of LiI. This has the added advantage of introducing a source of cations into the electrolyte at the same time although the source of cations and the redox promoter can be separate components. These cations can, in turn, be inserted into the electrochromic material. The following reactions depict the primary processes occurring during the coloration process in a system such as that just described.

$$
\begin{aligned}
& 3 \mathrm{I}^{-} \rightleftharpoons \mathrm{I}_{3}^{-}+2 \mathrm{e}^{-} \\
& \text {(transparent) } \mathrm{WO}_{3}+\mathrm{ne}^{-}+\mathrm{nLi}^{+} \rightleftharpoons \begin{array}{l}
\mathrm{Li}_{\mathrm{n}} \mathrm{WO}_{3} \\
\text { (colored) }
\end{array}
\end{aligned}
$$

In the above system, the removal of the applied DC potential to the device results in the return of the system to the highly transmitting state. This inherent self-bleaching mechanism upon an electrical failure has resulted in the commercialization of these devices in certain automotive applications ${ }^{3}$. Rapid self-bleaching generally also indicates high power consumption to maintain a desired level of 
coloration. High power consumption has resulted in difficulties in scaling the size due to the limited conductivity of commercially available transparent conductors. Methods have been suggested to reduce the currents necessary to sustain devices of this nature at desired levels of transparency.

\subsection{Notable new technologies}

There are two new technologies that have been introduced during the past year in the patent literature. These include the gasochromic and the user controlled photochromic devices. While there are no commercially available devices falling into these categories, they deserve mention based on their device structure and potential applications which overlap with the devices previously described.

\subsubsection{User controlled photochromic devices}

The other device of considerable interest to the electrochromic community is the user controlled photochromic device ${ }^{14,15}$. Traditional photochromic glass is characterized by a decrease in the transmission of the glass in the presence of light. Upon removal of such incident light, the glass regains the original high level of transmittance present before introduction to the light. It would be desirable to have photochromic devices that are capable of, at the discretion of the user, controlling transmission when exposed to radiation. The user controlled photochromic device allows for the decrease of light transmission upon exposure to radiation only if desired by the user. Additionally, these photochromic devices may be bleached to the original high transmissive state at the discretion of the user, even in the presence of radiation, by the application of an external electrical potential to the device.

The user controlled photochromic device ${ }^{14}$ allows a high degree of control over the level of photochromic activity of the glazing while in the presence of solar radiation. When the electrical leads of these devices are shorted, the device possesses a degree of photochromic activity. Examples shown by Teowee ${ }^{14}$ exhibit a 5:1 contrast ratio upon a 15 min exposure to typical solar radiation. Upon application of modest potentials between the transparent conductors, the device regains the original high level of transmission even in the presence of solar radiation. When the leads of the device are left in an open state, the photochromic activity is inhibited under the same solar exposure.

As shown in Fig. 5, a typical framework for the device is again two glass substrates with conductive transparent oxide coatings opposing one another. One conductor is coated with a light sensitive electrode $\left(e . g . \mathrm{TiO}_{2}\right)$ on one of the substrates which is pre-coated with a transparent conductive layer. The other conductor is coated with a chromogenic electrode. The cavity is then filled with an electrolyte containing at least one redox promoter and potentially a source of cations.

With the transparent conductors shorted, coloration proceeds in the presence of light. Redox reactions occurring at the surface of the radiation sensitive electrode result in both the generation of a potential across the device as well as the generation of electrons. This potential results in both the injection of cations from the electrolyte into the chromogenic electrode and the transport of electrons from the redox reaction into the chromogenic electrode through the external shorted leads. This double injection process results in coloration of the device.

Application of a modest external potential to the device results in removal of the cations and electrons from the chromogenic layer and thus results in a bleaching of the device.

While no commercial products are currently available which possess this technology, it holds great promise for future electrochromic glazings.

\subsubsection{Gasochromic}

Recent discussions of this type of technology have appeared in the literature with a good example found in

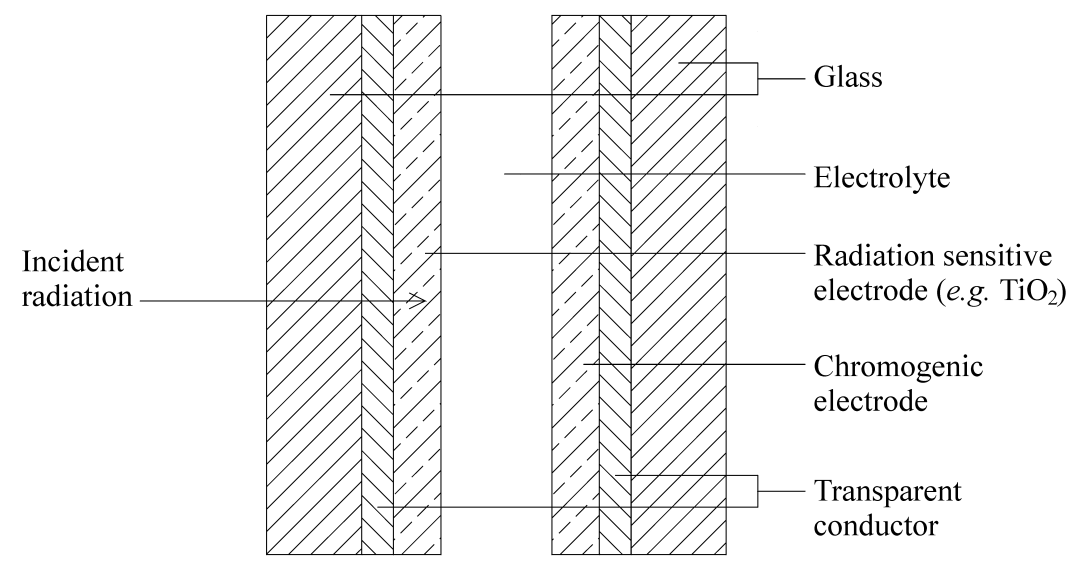

Figure 5. 


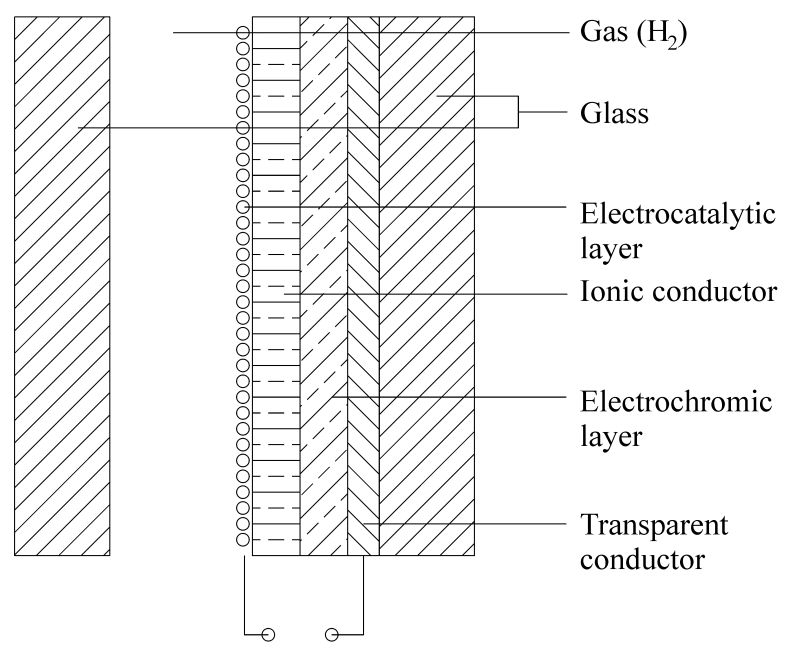

Figure 6.

reference ${ }^{16}$. The embodiment of the device is shown in Fig. 6. In essence, a gas such as hydrogen alone or mixed with inert carriers in cooperation with an electrocatalytic layer serves as the source of ions for insertion into an electrochromic electrode. Upon application of a potential between the electrocatalytic layer and the transparent conductor, the ions formed at the catalyst can be transported through an ionic conductor and into the electrochromic layer causing coloration of the device. Reversal of the potential results in the removal of the ions from the electrochromic electrode and a bleaching of the device. While the modulation of the exemplary device is only a modest $15 \%$ at $550 \mathrm{~nm}$ in about $2 \mathrm{~min}$, it does provide an alternative technology for electrochromic glazing.

\section{Current Components Used in Electrochromic Cells}

To make a large area device with the speed, transmission and modulation requirements for both automotive and architectural applications, several improvements will have to be made to the current device designs. Consistent to all devices are the transparent conductor films, which for large-area applications would require higher conductivity to enhance color kinetics and uniformity of coloration. The thin film electrochromic layers and ion-storage layers both anodically and cathodically coloring - could benefit from higher optical efficiencies and charge-storing capacities. The devices would preferably be solid state, which will necessitate good sealing of the devices. Finally, the device design should minimize costs of fabrication.

\subsection{Transparent conductors}

The most widely-used transparent conductors (TC) in EC devices are indium tin oxide (ITO) and fluorine- or antimony-doped tin oxide (FTO or ATO $)^{17}$. ITO for com- mercial applications is typically deposited by physical vapor deposition. Typically, FTO is deposited on the glass float line by chemical vapor deposition, while antimonydoped tin oxide is deposited by sputtering. FTO has superior temperature stability and under certain conditions has shown to be more electrochemically stable than $\mathrm{ITO}^{18}$. For these reasons it can be used as a substrate when producing EC coatings which require high temperatures $\left(>300{ }^{\circ} \mathrm{C}\right)$. One group has shown that passivation layers inserted between an electrochromic layer and the ITO layer can reduce degradation in the conductor in certain device configurations ${ }^{19}$. The least expensive transparent conductors which are currently available are FTO. These are available under the trade names "TEC" and "Sungate 500" from Libbey Owens Ford Industries (LOF) ${ }^{20}$ and Pittsburg Plate Glass $(\mathrm{PPG})^{21}$, respectively, and also from AFG Industries Inc. ${ }^{22}$ For large-area devices, it is required that the transparent conductor have a low sheet resistance, to reduce the voltage drop from the edges to the center of the device. For all practical sizes, the sheet resistance should be less than 20 ohms/sq.

It should be mentioned here that there are other TCs mentioned in the literature, such as aluminum-doped zinc oxide and fluorine-doped zinc oxide ${ }^{23}$. In addition, various groups have utilized the sol-gel process to produce TCs, but the conductivities of these films are markedly less than the commercial films ${ }^{24,25}$. Thin coatings of metal, such as gold, have also been used in this application ${ }^{16}$.

Ideally, for large area applications, transparent conductor deposition on the float line is preferred because of the low $\operatorname{cost}^{26}$.

\subsection{Cathodic films}

There are several excellent reviews on cathodic and anodic solid state electrodes which can be used in EC devices either as electrochromic or non-coloring ion storage layers ${ }^{27-30}$. We will restrict our discussion to materials suitable for large-area glazings. Of the cathodically-coloring materials, tungsten oxide is the most widely used and most cited in patent literature. This is because of its superior EC properties and relative ease of deposition by sputtering, evaporation, chemical vapor deposition and sol-gel. Evaporated $\mathrm{WO}_{3}$ has been used successfully in commercial EC products such as automotive mirrors. For large applications, wet chemical deposition methods or chemical vapor deposition on the glass float line might have a cost advantage in that they do not require large expensive vacuum controlled deposition chambers. The peroxo complexes of tungsten have been shown to be good precursors for wet chemical deposition ${ }^{31,32}$. Large-area coatings (35 $\mathrm{cm} \times 40 \mathrm{~cm}$ ) deposited by this method have been incorporated into devices which show a contrast ratio of 10:1 over several thousand color/bleach cycles ${ }^{33}$. 
Amorphous films of tungsten oxide are known to modulate their transmission primarily through absorption in the film. The crystalline material modulates some of the solar radiation by reflecting in the NIR region ${ }^{34}$. For energy-efficient EC glazings, materials with increasing modulation by reflection are preferred.

The color of tungsten trioxide changes from transparent to deep blue upon reduction. This blue color can be changed through the addition of other oxides or metals. In the case of $\mathrm{V}_{2} \mathrm{O}_{5}$ addition, the reduced film is green ${ }^{26}$, and when gold particles were added to a cermet of tungsten oxide ${ }^{35}$, the reduced material was red. Also of interest are "neutral"coloring films, which have a uniform response throughout the visible spectrum, giving the appearance of a gray, or more neutral color. Such behavior has been noted in the case of tungsten oxide films with molybdenum oxide as an additive $^{36}$.

$\mathrm{Nb}_{2} \mathrm{O}_{5}$ is reported to have EC properties which could rival those of $\mathrm{WO}_{3}$, although this material has yet to be used as a cathodic EC film in a patented device. It can be deposited by many methods including sputtering ${ }^{37}$, chemical vapor deposition ${ }^{38}$ and wet chemical ${ }^{39}$. Wet-chemical deposition of such coatings has been accomplished using $\mathrm{NbCl}_{5}$ as a precursor and is reported to have superior EC properties ${ }^{40}$. Lithiation of $\mathrm{Nb}_{2} \mathrm{O}_{5}$ through the addition of a lithium salt to the precursor solution enhances the EC kinetics - in particular the bleach rate and reversibility for lithium intercalation. This was shown for both the amorphous and crystalline material ${ }^{41}$. In comparison to $\mathrm{WO}_{3}$ niobium oxide requires higher potentials for color and bleach and has slower EC kinetics.

\subsection{Anodic coloring films and ion storage layers}

One of the materials most widely used as a counter electrode/anodically coloring film in patented electrochromic devices is iridium oxide $\left(\mathrm{IrO}_{2}\right)^{42,43}$. In a lithium ion based electrolyte, the $\mathrm{IrO}_{2}$ is a light brown color in the oxidized state, and becomes colorless when reduced. Common methods of depositing $\mathrm{IrO}_{2}$ are reactive sputtering ${ }^{44,45}$ and anodization ${ }^{46}$ of iridium metal. While iridium oxide has good properties regarding reversibility to lithium intercalation, it is an extremely expensive material.

An alternative to and less expensive material than $\mathrm{IrO}_{2}$, nickel oxyhydroxide has been prepared by numerous techniques, including sol-gel ${ }^{47}$. The properties of the sol-gel coatings were comparable to all other methods. $\mathrm{NiOOH}$ has been used in an all solid state thin film device involving the insertion of both protons and hydroxyl groups ${ }^{48,49}$. The $\mathrm{NiOOH}$ films are colorless in the reduced state, and become nearly black when oxidized.

Vanadium pentoxide colors anodically from its bright yellow oxidized state to nearly colorless when reduced. This film works best with a non-aqueous lithium ion electrolyte and has been used in devices as an ion storage layer rather than as the sole supplier of electrochromic response. $\mathrm{V}_{2} \mathrm{O}_{5}$ has a strong anodic coloration in the UV and visible regions and a weak cathodic coloration in the red and infrared. For this application $\mathrm{V}_{2} \mathrm{O}_{5}$ would not be a good "electrochromic" counter electrode to tungsten oxide, due to its modulation of the solar spectrum ${ }^{50}$. However, mixed oxides of vanadium and chromium or niobium ${ }^{51,52}$ are reported to significantly decrease or eliminate the modulation in the near infrared while maintaining a high charge capacity.

$\mathrm{TiO}_{2}$, known as a weak cathodic EC material, is modified easily by the addition of $\mathrm{CeO}_{2}$ to produce a colorless mixed oxide. The addition of $\mathrm{CeO}_{2}$ is said to "quench" the color reaction in the $\mathrm{TiO}_{2}$, and, depending on the ratio of $\mathrm{CeO}_{2}$ to $\mathrm{TiO}_{2}$, increase the charge capacity of the film ${ }^{53}$. The addition of $\mathrm{TiO}_{2}$ is also reported to enhance the intercalation kinetics of sol-gel derived $\mathrm{CeO}_{2}{ }^{54,55}$.

\subsection{Electrolytes}

A major component of all electrochromic devices mentioned previously is the transparent ionic conductor. It is necessary not only for the function of the electrochemical processes occurring within the device but it is also necessary for the mechanical properties. Use of liquid electrolytes has been acceptable in some small electrochromic devices, but as the dimensions of the electrochromic glazing increase the hydrostatic pressure begins to put excessive stresses on the electrodes and seals. Bowing of the glass panes in the device ultimately results in non-uniformity of the electrolyte thickness and resistivity as well resulting in unwanted cosmetic effects even if the mechanical integrity is maintained. Recent reviews of semi-solid and solid electrolytes in electrochromic devices appear in Electrochromism: Fundamentals and Applications ${ }^{30}$ and Handbook of Inorganic Electrochromic Materials ${ }^{27}$.

Many systems have attempted to address this issue by use of various thickened electrolytes consisting of a major solvent system, ionic salt, and an intermediate molecularweight polymer. This type of electrolyte is attractive for device applications due to the ease of preparation and the ability to reduce many of the unwanted effects of a strictly liquid electrolyte. Ideally, however, a more rigid electrolyte is desirable. While a rigid electrolyte can be achieved with high molecular-weight polymer additions, it can lead to great difficulty during filling and assembly of devices by requiring high pressures and or temperatures to achieve proper flow for rapid and uniform assembly.

There are a number of examples of solid electrolytes in electrochromic devices which appear in the recent patent literature. While perhaps the most direct approach to the preparation of solid electrolytes is the deposition of inorganic films by sputtering or evaporation ${ }^{56,57}$ or by sol-gel ${ }^{58,}$ ${ }^{59}$, this method presents difficulty in assembly of complete device structures. Once the electrolyte is deposited in this 
manner, subsequent layers are typically deposited by sputtering or evaporation as well. Thus the device is built upon a single glass substrate, and the final layer deposited is a conductor being either a metallic reflective layer or a transparent conductor. While the fabrication of such a device is attractively simple, one must then address the issues of providing hermetic sealing, pre-reduction of electrodes if necessary, and protection for these layers.

A preferred method of forming solid electrolytes for electrochromic devices is the in situ gelling of a liquid electrolyte. In this approach, the device electrodes are prepared with their respective coatings and assembled with sealing materials to form a cavity for the electrolyte. A liquid electrolyte is then injected into the device where it is solidified. Application of such techniques to the electrochemichromic system are described by Varaprasad ${ }^{60}$ and Tonar $^{61}$ where the anodic and cathodic coloring compounds are incorporated in a polymeric electrolyte with potentially some inorganic material. Crosslinking takes place within the electrolyte upon exposure to heat or ultraviolet radiation.

The in situ polymerization has also been reduced to practice in systems with electrolytes employed in ion storage electrochromic systems. One literature example ${ }^{62} \mathrm{de}-$ scribes a device based on glycidoxypropyltrimethoxysilane and tetraethoxysilane with addition of ionic salt and a zirconium alkoxide. This electrolyte could be injected into the cell and cross-linked by heating to $100{ }^{\circ} \mathrm{C}$. Although the system has a modest ionic conductivity of $10^{-4}$ to $10^{-5} \mathrm{~S} \mathrm{~cm}^{-1}$, the advantages of an adhesive electrolyte which doesn't flow with time and is integral to the structural stability of the device make these types of systems very attractive for large area applications.

\section{Summary}

To date, even though there are many promising technologies, there is no commercially-available large-scale EC glazing. In terms of EC devices with solution phase redox materials, significant improvements have been made in terms of solidifying the electrolyte and reduction of segregation. Devices involving a solid-state electrochromic layer have also seen improvement in the areas of a solid electrolyte, ion-storage layers and electrochromic films. Introduced in the last year, an exciting new device configuration known as the user-controlled photochromic device promises the ability to produce large-area devices with low power consumption. Despite the tremendous advances in the art, it appears that significant advances in terms of scalability, cost and device performance must be made before commercial applications can be realized.

\section{References}

1. Warner, J.L.; Reilly, M.S.; Selkowitz, S.E.; Arasteh, D.K.; Ander, G.D. Utility and Economic Benefits of
Electrochromic Smart Windows, Lawrence Berkeley Laboratories (LBL)-32368, 1992.

2. Lampert, C.M. Sol. Energy Mater. Sol.Cells, v. 52, p. 207, 1998.

3. Lynam, N.R.; Agrawal, A. Large Area Chromogenics: Materials \& Devices for Transmittance Control, Lampert C.M.; Granquist, C.G., eds., SPIE, Optical Engineering Press, Bellingham, Washington, p. 46, 1989.

4. Varaprasad, D.V.; Lynam, N.R.; Habibi, H.R.; Desaraju, P. U.S. Patent 5,725,809, 1998.

5. Byker, H.J.; Srinivasa, R. U.S. Patent 5,770,114, 1998.

6. Byker, H.J. U.S. Patent 5,751,467, 1998.

7. Van Dine, J.E.; Parkhe, V.D.; Klein, L.C.; Trumbore, F.A. U.S. Patent 5,699,192, 1997.

8. Lefrou, C.; Defendini, F.; Garot, F.; Marrot, O. U.S. Patent 5,663,829, 1997.

9. Ellis Jr., F.B.; Van Dine, J.E.; Parkhe, V.D. U.S. Patent 5,757,537, 1998.

10. Defendini, F. U.S. Patent 5,379,146, 1995.

11. Nagai, J.; Seike, T. U.S. Patent 5,721,633, 1998.

12. Varaprasad, D.V.; Habibi, H.; McCabe, I.A.; Lynam, N.R. U.S. Patent 5,668,663, 1997.

13. Kamimari, T.; Nagai, J.; Mizuhashi, M. U.S. Patent 4,671,619, 1987.

14. Teowee, G.; Allemand, P.-M.; Cronin, J.P.; Tonazzi, J.C.L.; Agrawal, A. U.S. Patent 5,604,626, 1997.

15. Teowee, G.; Gudgel, T.J.; McCarthy, K.; Agrawal, A.; Allemand, P.M.; Cronin, J.P. To be published in Electrochim. Acta, Proc. of International Meeting on Electrochromics.

16. Wittwer, V.; Georg, A.; Graf, W. European Patent WO 98/06000, 1998.

17. Lynam, N.R. Proc. on Electrochromic Mat., Carpenter, M.K.; Corrigan D.A., eds., Physical Electrochemistry Division, The Electrochemical Society, Pennington, New Jersey, 90-2, 201, 1990.

18. Radhakrisnan, S.; Unde, S.; Mandale, A.B. Mat. Chem. Phys., v. 48, p. 268, 1997.

19. Boulanger, F.; Lerbet, F.; Papret, C.; Perrodo, F.; Thomas, G. U.S. Patent 5,352,504, 1994.

20. Libbey Owens Ford, Pub. No. 191 TEC 157-1, Rev., 1991.

21. Pittsburgh Plate Glass Tech. Rep. n. Sgt 5-1, 1993.

22. AFG Industries, Inc., USA, Comfort E and E2 Glass.

23. Ohyama, M.; Kozuka, H.; Yoko, T. J. Am. Ceram. Soc., v. 81, p. 1622, 1998.

24. Mattox, D.M. Thin Solid Films, v. 204, p. 25, 1991.

25. Djaoued, Y.; Phong, V.H.; Badilescu, S.; Ashrit, P.V.; Girouard, F.E.; Truong, V.-V. Thin Solid Films, v. 293, p. 108, 1997. 
26. McCurdy, R.J.; Soubeyrand, M.J. Chemical Vapor Deposition. Proceedings of the Fourteenth International Conference and EUROCVD-11, 463, 1997.

27. Granqvist, C.G. Handbook of Inorganic Electrochromic Materials, Elsevier Science Publishers, B.V., Amsterdam, 1995.

28. Lev, O.; Wu, Z.; Bharathi, S.; Glezer, V.; Modestov, A.; Gun, J.; Rabinovich, L.; Sampath, S. Chem. Mater., v. 9, p. 2354, 1997.

29. Agrawal, A.; Cronin, J.P.; Zhang, R. Sol. Energy Mater. Sol. Cells, v. 31, p. 9, 1993.

30. Monk, P.M.S.; Mortimer, R.J.; Rosseinsky, D.R. Electrochromism: Fundamentals and Applications, VCH, Weinheim, 1995.

31. Kudo, T.; Okamoto, H.; Matsumoto, K.; Sasaki, Y. Inorg. Chim. Acta, v. 111, L27, 1986.

32. Cronin, J.P.; Tarico, D.J.; Agrawal, A.; Zhang, R. U.S. Patent 5,277,986, 1994.

33. Cronin, J.P.; Kennedy, S.R.; Agrawal, A.; Gudgel, T.J.; Yao, Y.J.; Tonazzi, J.C.L.; Uhlmann, D.R. To be published in Electrochemistry of Glass and Ceramics, Ceramic Transactions, v. 92.

34. Cogan, S.F.; Plante, T.D.; Parker, M.A.; Rauh, R.D. Sol. Energy Mater. Sol. Cells, v. 14, p. 185, 1986.

35. Sichel, E.K.; Gittleman, J.I. Appl. Phys. Let., v. 33, p. 564, 1978.

36. Monk, P.M.S.; Chesler, S.L. Electrochim. Acta, v. 38, p. $1521,1993$.

37. Cogan, S.F.; Anderson, E.J.; Plante, T.D.; Rauh, R.D. Proc. Soc. Photo-Opt. Instrum. Eng., 562, 23, 1985.

38. Maruyama, T.; Kanagawa, T. J. Electrochem. Soc., v. 141, p. 2868, 1994.

39. Faria, R.C.; Bulhoes, L.O. J. Electrochem. Soc., v. 141, L29, 1994.

40. Avellaneda, C.O.; Macedo, M.A.; Florentino, A.O.; Aegerter, M.A. Proc.SPIE, 2255, 38, 1994.

41. Macek, M.; Orel, B.; Krasovec, U.O. J. Electrochem. Soc., v. 144, p. 3002, 1997.

42. Niwa, T.; Uchikawa, K.; Endo, T. Japan Displays, P2.19, 372, 1986.

43. Dautremont-Smith, W.C. Display, v. 4, p. 67, 1982.
44. Schiavone, L.M.; Dautremont-Smith, W.C.; Beni, G.; Shay, J.L. Apply Phys. Lett., v. 34, p. 823, 1979.

45. Shay, J.L.; Beni, G. IEEE Trans. Electron. Dev., v. 26, p. $1138,1979$.

46. Rice, C.E.; Bridenbaugh, P. Appl. Phys. Lett., v. 38, p. $59,1981$.

47. Surca, A.; Orel, B.; Pihlar, B. J. Sol-Gel Sci. Tech., v. 8, p. 743, 1997.

48. Nemetz, A.; Temmink, A.; Bange, K.; de Torresi, S.C.; Gabrielli, C.; Torresi, R.; Hugot-Le Goff, A. Sol. Energy Mater. Sol. Cells, v. 25, p. 93, 1992.

49. Moser, F.H.; Lynam, N.R. U.S. Patent 4,959,247, 1990.

50. Cogan, S.F.; Nguyen, N.M.; Perrotti, S.J.; Rauh, R.D. J. Electrochem. Soc., v. 57, p. 1016, 1988.

51. van Dine, J.E. U.S. Patent 5,404,244, 1995.

52. Cogan, S.F.; Rauh, R.D.; Nguyen, N.M.; Plante, T.D.; Westwood, J.D. J. Electrochem. Soc., v. 140, p. 112, 1993.

53. Kullman, L.; Veszelei, M.; Ragan, D.D.; Isidorsson, J.; Vaivars, G.; Kandars, U.; Azens, A.; Schelle, S.; Hjorvarsson, B.; Granqvist, C.G. Proc. SPIE, v. 209, p. $2968,1997$.

54. Tonazzi, J.C.L.; Valla, B.; Macedo, M.A.; Baudry, P.; Aegerter, M.A.; Rodriguez, A.C.M.; Bulhoes, L.O. Proc. SPIE, v. 375, p. 1328, 1990.

55. Baudry, P.; Rodriguez, A.C.M.; Aegerter, M.A.; Bulhoes, L.O. J. Non-Cryst. Solids, v. 121, p. 319, 1990.

56. Hashimoto, S.; Terada, J. U.S. Patent 5,777,779, 1998.

57. Nagai, J.; Seike, T. U.S. Patent 5,721,633, 1998.

58.Zhang, J.; Benson, D.; Tracy, C.E. U.S. Patent 5,716,736, 1998.

59. Van Dine, J.; Parkhe, V.D.; Klein, L.C.; Trumbore, F.A. U.S. Patent 5,659,417, 1997.

60. Varaprasad, D.; Agrawal, A.; Zhao, M.; Allemand, P.; Dornan, C.; Lynam, N. EP 0612 826, A1, 1994.

61. Tonar, W.L.; Anderson, J.S.; Theiste, D.A. U.S. Patent 5,679,283, 1997.

62. Munro, B.; Kramer, S.; Zapp, P.; Krug, H.; Schmidt, H. Proc. SPIE, 3136, 470, 1997. 